\title{
Damping of Coupled Bunch Instabilities by rf Frequency Modulation
}

\author{
Bruno Zotter
}

October 18,2002

\section{Introduction}

In many high-energy synchrotrons and storage rings operating with bunched beams, instabilities occur due to coupling from bunch to bunch by wake fields in the surrounding vacuum chamber structures. The most direct cure is to reduce the strength of the wake fields by changing the structures which cause the strongest wakes, e.g. damping of higher modes (HOMs) in rf and/or incidental cavities, such as kicker tanks, or sleeving (shielding) of unavoidable cross section variations such as bellows or pumping T's. A time honored method is to simply change the temperature of the cooling water which may change the frequency of the resonant modes in a cavity sufficiently to avoid coupling to an unstable beam mode of oscillation. However, in many instances these methods are insufficient and other measures have to be used, such as feedback systems. However, for machines with a large number of bunches such systems usually need to have a rather broad bandwidth and also require costly space around the machine circumference.

It is often simpler to rely on reducing the coupling between bunches by other means. One method is to vary the distance between bunches - e.g. introducing gaps of different lengths by leaving out a certain number of bunches from the regularly spaced "buckets" formed by the rf voltage. However, uneven spacing may have disadvantages - in particular for colliders where the bunches should meet the opposing beam at the fixed locations of detectors. Therefore it is often preferable to reduce coupling by varying the natural oscillation frequencies of the bunches. 
For longitudinal oscillations, the natural frequency of oscillation of a bunch is its coherent synchrotron frequency $\omega_{s}$. It changes very little with the number of particles in a bunch, hence their variation would be inefficient. However, $\omega_{s}$ is proportional to the square root of the rf voltage, and a modulation of the voltage seen by each bunch is thus often sufficient to inhibit an instabilitity. This technique has been applied already many years ago, e.g. at ADONE in Frascati (quoted in [1], in the CERN PS[2], at DORIS at DESY[3] and in the Fermilab booster[4], where a voltage modulation was obtained by simply changing the harmonic number of one or two rf cavities. To my knowledge, the first quantitative analysis was published by Dieter Moehl[1] and provided a "decoupling criterion". A number of papers followed $[5,6]$ which supplemented - and partially contradicted - these early findings as will be discussed below.

For transverse bunch oscillations, the coherent betatron frequency changes with the number of particles in a bunch, and hence a variation of the bunch currents sometimes has the desired effect. However, this is often not acceptable for other reasons, and also may be too weak. It can then be supplemented or replaced by other means such as a radio frequency quadrupole (RFQ), which method has been used successfully in DORIS[3]. Nowadays feedback systems are most often used to stabilize transverse bunch oscillations as wider bandwidths have become accessible with digital techniques.

\section{The decoupling criterion}

In order to estimate the required modulation depth for a given strength of wake fields, the analysis in ref.[1] starts from the linearized equation of motion describing the oscillations of a characteristic quantity, e.g. the phase displacement from the equilibrium position for the longitudinal dipole mode, or the bunch length for the corresponding quadrupole mode. Assuming a train of $n$ bunches, the equation of motion for the $\ell$-th bunch are written

$$
\frac{d^{2} x_{\ell}}{d t^{2}}+\omega_{\ell}^{2} x_{\ell}=2 \bar{\omega} \sum_{m=1}^{n} W_{\ell m} x_{m}
$$

where $\omega_{\ell}$ is the (coherent) synchrotron frequency of the $\ell$-th bunch and $W_{\ell m}$ the wake field of the $\mathrm{m}$-th bunch acting on the $\ell$-th one. The average frequency $\bar{\omega}$ is introduced such that the "wake fields", which are actually "dispersion relation coefficients" [9] $W=U+i V$, have the dimensions of a fre- 
quency $^{1}$. Searching for exponential solutions proportional to $\exp (i \lambda t)$, one obtains a system of $n$ homogeneous linear equations $\sum M_{\ell m} x_{m}=0$ with $\ell, m=1,2, . . n$, where

$$
M_{\ell m}=\left\{\begin{array}{ll}
W_{\ell m} & \text { for } m \neq \ell, \\
W_{\ell \ell}-\omega_{\ell}^{2}+\lambda^{2} & \text { for } m=\ell .
\end{array}\right\}
$$

Solutions of such a system of equations are only possible if its determinant vanishes, i.e. $\operatorname{det} M_{\ell m}=0$ or

$$
\operatorname{det}\left[2 \bar{\omega} W_{\ell m}-\delta_{\ell m}\left(\omega_{\ell}^{2}-\lambda^{2}\right)\right]=0
$$

The eigenvalues $\lambda$ are usually quite close to the natural frequencies $\omega_{\ell}$, therefore $\omega_{\ell}^{2}-\lambda^{2} \approx 2 \bar{\omega}\left(\omega_{\ell}-\lambda\right)$ and one obtains the simpler condition

$$
\operatorname{det}\left[W_{\ell m}-\delta_{\ell m}\left(\omega_{\ell}-\lambda\right)\right]=0
$$

A spread in the diagonal wake fields $W_{\ell \ell}$ is equivalent to a spread of the frequencies $\omega_{\ell}$ and thus need not be studied separately.

First the author investigates the case of short range wakes, extending only to the next bunch, i.e. $W_{\ell m}=0$ except when $m=\ell+1$. Introducing the average wake field $\bar{W}=\left(\Pi_{\ell=1}^{n} W_{\ell+1, \ell}\right)^{1 / n}$ one obtains the characteristic equation which determines $\lambda$

$$
\Pi_{\ell=1}^{n}\left(\omega_{\ell}-\lambda\right)=\bar{W}^{n},
$$

with the solutions $\lambda=\omega_{\ell}-\bar{W} \exp (2 \pi i \ell / n)$. One sees that a spread of the wake fields will not strongly influence the result which depends only on their geometrical average.

Next assume that the synchrotron frequency has a sinusoidal modulation from bunch to bunch

$$
\omega_{\ell}=\bar{\omega}+\Delta \omega \cos \left(\frac{2 \pi \ell}{n}+\phi\right)
$$

where $\phi$ is the phase of the modulation which can be chosen. For $\phi=\pi / n$ only half the bunches have different frequencies, while for $\phi=\pi / 2 n$, they

\footnotetext{
${ }^{1}$ In the original definition, only the transverse dispersion relation coefficients had the dimension of inverse time[9], while the longitudinal ones were defined as energies; converting them to frequencies needs additional scaling
} 
are all different. It is expedient to introduce dimensionless quantities

$$
\begin{aligned}
\alpha_{\ell} & =\frac{\omega_{\ell}-\bar{\omega}}{\Delta \omega}=\cos \left(\frac{2 \pi \ell}{n}+\phi\right), \\
\Delta & =\frac{\lambda-\bar{\omega}}{\Delta \omega}=\cos \theta,
\end{aligned}
$$

Then one obtains the equation

$$
\Pi_{\ell=1}^{n}\left(\alpha_{\ell}-\Delta\right)=\Pi_{\ell=1}^{n}\left[\cos \left(\frac{2 \pi \ell}{n}+\phi\right)-\cos \theta\right]=\left(\frac{\bar{W}}{\Delta \omega}\right)^{n} .
$$

The finite product over $\ell$ can be converted, using Eq.1.395.1 of ref[8] ${ }^{2}$, to get for $\phi=\pi / n$

$$
\Pi_{\ell=1}^{n}\left(\alpha_{\ell}-\Delta\right)=\frac{1}{2^{n-1}}[\cos (n \phi)-\cos (n \theta)]
$$

and thus

$$
\cos (n \theta)=\cos (n \phi)-\left(\frac{-2 \bar{W}}{\Delta \omega}\right)^{n} .
$$

For the usual case $|\bar{W}| \ll \Delta \omega$, one obtains the "decoupling criterion" for short range wakes

$$
\Delta \omega \geq 2|\bar{W}|
$$

which is claimed to be sufficient for a large reduction of the growth rate of the coupled bunch instability.

For the case of long range wakes, which couple all bunches together ("high Q case"), the analysis leads to the condition

$$
\sin \theta \cot \left(\frac{n}{2} \theta\right) \leq-\frac{\bar{W}}{\Delta \omega} .
$$

When one assumes $|\bar{W}| \ll \delta \omega$ and expands in $\delta \omega$, convergence is obtained only under the additional condition $|W| / \Delta \omega \ll 2 / n$. Then one finds a reduction of the growth rate $1 / \tau$ by a factor $3 / n$.

If the number of bunches is large, $n \gg 1$, this additional condition is often too stringent, but then the approximation $\cot (n \theta / 2) \approx-i$ can be used to get another form of the "decoupling criterion"

$$
\Delta=\sqrt{1+\left(\frac{\bar{W}}{\Delta \omega}\right)^{2}} .
$$

\footnotetext{
${ }^{2}$ correcting a mis-print in Eq.(14) of the original paper - the factor $2^{(n-1)}$ is on the wrong side
} 
For $|\bar{W}| \ll \Delta \omega$, the growth rate will then be reduced by a factor $|\operatorname{Re} \bar{W}| / \Delta \omega$. For $\phi=\pi / n$ the same equations hold with $n / 2$ replaced by $n$.

In an existing machine, the imaginary part of $V=\operatorname{ImW}$ can be found from the growth rate of an instability since $V=1 / \tau$. The imaginary part is related to the incoherent synchrotron frequency shift with current which is more difficult to measure. For machines with a dominant resistive wall impedance $U \approx V$, and hence $|W|=\sqrt{U^{2}+V^{2}}=V \sqrt{2}$. Solution of Eq.(13) then permits the calculation of the growth rate for a given modulation depth.

\section{Stability region for sinusoidal modulation}

Many years after the original report, the problem of damping a multi-bunch instability by a bunch to bunch frequency spread was analyzed at DESY in the framework of Landau damping[6]. There transverse motion was studied, including kicks due to transverse wake fields, summing them over all other bunches and all previous turns. With the longitudinal position $s$ as independent variable, the equation of motion of the $\ell$-th bunch in a total of $n$ bunches can be written

$$
\frac{d^{2} y_{\ell}}{d s^{2}}+k_{\ell}^{2} y_{\ell}=\frac{e^{2}}{2 \pi R E} \sum_{m=-\infty}^{\infty} \sum_{j=0}^{n-1} Q_{j} W_{\perp}\left(t_{\ell}-t_{j}\right) y_{j}(s-2 \pi R m),
$$

where $k_{\ell}$ is the betatron wave number in a storage ring with circumference $2 \pi R, y_{j}$ the vertical displacement and $Q_{j}$ the charge of the $\mathrm{j}$-th bunch ${ }^{3}$ Due to causality, the (transverse) wave function $W_{\perp}(t)$ must vanish for negative arguments, and thus the summation over $m$ could be extended over all turns from $-\infty$ to $+\infty$. For equal spacings, the time interval between bunches is

$$
t_{m}-t_{\ell}=\left(\frac{m-\ell}{n}+k\right) T
$$

where $T$ is the revolution time. Introducing the impedance $Z_{\perp}$ as the Fourier transform of the wake function. and looking for exponential solutions of the equation of motion, one again obtains a set of simultaneous, homogeneous, linear equations. A solution can be found only if the determinant $\operatorname{det}(I-M)$

\footnotetext{
${ }^{3}$ The dimensions of the factor used on the RHS are only correct if $Q_{j}$ is the normalized charge $Q / e$, and $W$ the wake function per unit length, i.e. $W_{\perp} / 2 \pi R$; we also use the energy $E=\gamma m_{0} c^{2}$.
} 
vanishes, where the matrix elements for equally populated bunches $Q_{\ell}=Q$ are given by

$$
M_{q p}=i \frac{e^{2} Q R}{2 \pi E T} Z_{\perp}\left[(p+\nu) \omega_{0}\right] \sum_{\ell=0}^{n-1} \frac{1}{\nu_{\ell}^{2}-\nu^{2}} \exp \left[2 \pi i(q-p) \frac{\ell}{n}\right]
$$

where $\nu$ is the oscillation tune to be determined.

For a single, sharp impedance the determinant reduces to the single term for which $(p+\nu) \omega_{0}$ is close to the resonant frequency. One gets the characteristic equation

$$
1=\frac{i e^{2} R}{2 \pi E T} Z_{\perp}\left[(p+\nu) \omega_{0}\right] \sum_{\ell=0}^{n-1} \frac{Q_{\ell}}{\nu_{\ell}^{2}-\nu^{2}}
$$

A distribution of tunes around the unperturbed $\nu_{0}$ value may be written

$$
\nu_{\ell}=\nu_{0}+\Delta \nu \frac{x_{\ell}}{2}
$$

where $-1<x_{\ell}<1$ is determined by the form of the modulation. For $\Delta \nu \ll \nu_{0}$, one may approximate $\nu_{\ell}^{2}-\nu^{2} \approx 2 \nu_{0}\left(\nu_{\ell}-\nu\right)$ and introduce the (dimensionless) transverse "dispersion relation coefficients"

$$
U+i V=-i \frac{e^{2} Q R}{E T} Z_{\perp}\left[(p+\nu) \omega_{0}\right] \frac{n}{2 \nu_{0} \Delta \nu}
$$

to obtain the characteristic equation

$$
1=-(U+i V) \frac{\Delta \nu}{n} \sum_{\ell=0}^{n-1} \frac{1}{\nu_{\ell}-\nu} .
$$

One may replace the summation by an integration, which is easier to handle analytically, when the oscillation tune $\nu$ is further removed from the real axis than the spacing between neighboring bunch tunes $\nu_{\ell}$. This condition can be written $\operatorname{Im} \nu>\Delta \nu / n$, i.e. it is of interest for fast growth rates and is most easily fulfilled for a large number of bunches.

The dispersion relation for a sinusoidal modulation with harmonic one then becomes, with $x_{1}=2\left(\nu-\nu_{0}\right) / \Delta \nu$ :

$$
1=-(U+i V) \frac{1}{\pi} \int_{-\pi}^{\pi} \frac{d x}{\sin x-x_{1}} .
$$


The integration yields

$$
1=-(U+i V)\left\{\begin{array}{ll}
\frac{2 i}{\sqrt{x_{1}^{2}-1}} & \text { for }\left|\operatorname{Re} x_{1}\right|>1 \\
\frac{2 i}{\sqrt{1-x_{1}^{2}}} & \text { for }\left|\operatorname{Re} x_{1}\right|<1
\end{array}\right\}
$$

The stability limit $\operatorname{Im} x_{1}=0$ consists of 2 coincident lines, up and down the $\mathrm{V}$-axis, and has thus zero area. Therefore there is no stability possible for sinusoidal modulation with harmonic one in a single resonator impedance.

This does not directly contradict the former findings, where actually only a reduction of the growth rate was obtained. However, it strengthens the case for the use of other than sinusoidal modulation with harmonic one, e.g. using a higher harmonic. In the conclusions, the paper recommends fractional filling to fight multi-bunch instabilities.

A second DESY report from the same period[3] discusses the effect of frequency modulation on the required bandwidth of a feedback system. Usually the bandwidth needed is essentially given by the spacing between bunches and can thus become quite large for multi-bunch storage rings. However, if the bunch frequencies are split by a modulation, the required bandwidth is given by its frequency width and thus may often be drastically reduced. In addition, a barycentric feedback will then also damp higher modes which are thereby coupled to the lowest mode.

Due to a frequency splitting, each bunch "sees" essentially only the signal from to its own motion, while the signals of all other bunches average out. It is thus important that all bunches have different frequencies and not two of them are alike. Then the maximum damping rate can be estimated by

$$
\delta_{r}=\frac{\Delta \omega}{2 S(n)},
$$

where $\Delta \omega$ is the frequency difference between bunches, and $S(n)=\sum_{j=1}^{n-1} 1 / j$ which for large $n$ can be approximated by $S(n)=C+\log (n-1)$ where $C \approx 0.577$ is Eulers constant. Finally, experimental verification of these results at the storage ring PETRA is also reported there. 


\section{Application to Fermilab machines}

The 8 rf cavities in the Tevatron collider are arranged such that 4 of them accelerate protons in one direction and 4 anti-protons in the other. This is achieved by locating them $3 / 4$ wave lengths apart, and phasing them with $\pm \pi / 2$ phase shift. In this manner actually 6 cavities accelerate in one direction, while the other 2 decelerate, and similar in the opposite sense.

The exact distance between cavities is thus related to their wave length (or frequency), and even a small modulation would destroy this delicate balance. We conclude therefore that a frequency modulation by changing the rf harmonic of one or more cavities in the Tevatron collider is not possible without a complete redesign of the rf system. However, a longitudinal feedback system or "damper" has recently been completed and it is hoped that it will be capable of keeping the bunches stable.

The situation is different in the Fermilab Booster, where 18 cavities are used to accelerate protons in just one direction. For operation with high currents, a longitudinal coupled-bunch instability occurs which dilutes the beam. Already many years ago[4] it has been counteracted by detuning rf cavities. Tuning 2 of $n$ cavities from frequency $h \omega_{0}$ down to $(h-1) \omega_{0}$ yields

$$
\begin{aligned}
V_{1} & =(n-2) V \cos \left(h \omega_{0} t\right)+2 \alpha V \cos \left[(h+1) \omega_{0} t\right] \\
& =V\left\{\cos \left(h \omega_{0} t\right)\left[(n-2)+\alpha \cos \left(\omega_{0} t\right)\right]+\alpha \sin \left(\omega_{0} t\right) \sin \left(h \omega_{0} t\right)\right\}
\end{aligned}
$$

where $\alpha$ describes the reduction of rf voltage when a cavity is detuned. The actual reduction depends on the bandwidth of the cavity, and the detuning is efficient only it is large enough to keep a sufficient voltage at the harmonic sideband. Tuning one cavity up and another one down by one harmonic each results in a cleaner modulation of the rf frequency with the first harmonic or revolution frequency

$$
\begin{aligned}
V_{2} & =(n-2) V \cos \left(h \omega_{0} t\right)+\alpha V \cos \left[(h+1) \omega_{0} t\right]+\alpha V \cos \left[(h-1) \omega_{0} t\right] \\
& =V \cos \left(h \omega_{0} t\right)\left[(n-2)+2 \alpha \cos \left(\omega_{0} t\right)\right] .
\end{aligned}
$$

Since the booster cavities have a wide tuning range required for proton acceleration, we may assume $\alpha \approx 1$. Hence the modulation depth is about $2 \alpha /(n-2)$ or nearly $10 \%$. If necessary, the modulation depth can be easily increased by detuning more than 2 cavities. Picture of the rf voltage corresponding to the Fermilab booster parameters ( $n=18$ and $h=84)$ are shown 
in Fig. 1 for the case of 2 cavities tuned down, and Fig. 2 when one cavity is tuned down and one up by one harmonic number.

The desired modulation depth can be estimated from the frequency shift obtained from Moehl's "detuning criterion" [1] for long range wakes. In the CERN PS with 20 bunches, it was found one needed approximately four times the growth rate of the instability, $\Delta \omega \approx 4 V=4 / \tau$. Unfortunately, the growth rate of the longitudinal coupled bunch oscillations in the Fermilab booster have not been given in ref[4], but the picture shows of a large oscillation occurring $3 \mathrm{~ms}$ before extraction. Estimating the growth time $\tau$ to be of the same order of magnitude, one only needs a frequency shift of only $4 / \tau=130 s^{-1}$ to reduce the growth rate so it no longer dilutes the beam before extraction.

For the proposed Fermilab proton driver, the situation would be similar to that in the booster. There are 20 cavities foreseen, rather than only 18 , and their tuning range is somewhat smaller as injection is at higher energy (600 instead of $400 \mathrm{MeV}$ ). Hence they are tunable from 42.1 to $52.8 \mathrm{MHz}$, corresponding to the 84-th harmonic as the energy increases to $8 \mathrm{GeV}$. Tuning down to the 83-rd harmonic at top energy, corresponding to about 51.9 $\mathrm{MHz}$, is thus no problem. Tuning up to the 85-th harmonic is outside the normal tuning range and - depending on the quality factor of the cavity might reduce the voltage $(\alpha<1)$. Detuning of a single cavity reduces the modulation depth, but one can detune several cavities downwards.

Since the projected beam current is considerably higher than in the booster, coupled bunch instabilities will have faster growth rates and need more modulation to reduce it to acceptable values. The resistive wall effect was estimated to cause a transverse coupled bunch instability with a growth time of $3.3 \mathrm{~ms}[10]$. However, the effects of the higher modes in the rf cavities, which may cause both longitudinal and transverse coupled bunch instabilities, are more difficult to estimate as their exact frequencies can not be known to sufficient accuracy. However, as the beam accelerates, its harmonics move rapidly through any fixed higher-order modes, which thus remain dangerous only for the last few ms when the frequency change is small. Hence one has to bring the growth rate down to the order of a fraction of one ms to avoid large oscillation amplitudes which could lead to large oscillations and subsequent emittance dilution. Only a narrow-band longitudinal feedback system is necessary, of the order of the frequency modulation[3].

Application of the detuning method to the Fermilab Main Injector is somewhat different. This machine accelerates protons (or anti-protons) from 
8 to $150 \mathrm{GeV}$, and the rf frequency operating on the 588-th harmonic, changes only from 52.8 to $53.1 \mathrm{MHz}$. As the frequency range of its rf-system can be limited to less than $300 \mathrm{KHz}$, the cavities need not be tunable but need only have sufficient bandwidth. Since in this large ring the revolution frequency is only about $95 \mathrm{KHz}$, detuning at top energy by plus or minus one harmonic should be well inside this cavity bandwidth. However, due to the higher beam current, the coupled bunch instabilities driven by higher modes in the cavities might have rather short growth times and need to be damped either by mode-dampers in the cavities, or by a feedback which could again have reduced bandwidth if the synchrotron frequencies of the bunches are modulated.

\section{Conclusions}

We discussed several means of damping coupled-bunch instabilities in the Tevatron collider and its injectors. In particular, we have studied a number of publications treating stabilization of longitudinal oscillations by modulating the synchrotron frequencies from bunch to bunch. Some of these papers come to slightly different conclusions which appear contradictory at first sight, but are actually due to the limitation of these methods to only reduce the growth rates rather than completely stabilize a beam. In particular, a spread in the synchrotron frequencies can be obtained simply by detuning of one or several rf cavities which was envisaged for improving the operation several of the rings used in run-II.

Unfortunately, detuning of rf cavities is not applicable to the Tevatron collider itself due to the particular arrangement of the cavities there which have to accelerate counter-rotating beams. However, this method could be used in the Fermilab booster, where it has already been tried many years ago with limited success. We apply Moehl's "decoupling criterion" to show that detuning of 2 cavities in opposite direction will reduce the growth rate of the oscillations, but complete stabilization cannot be obtained unless other means are applied simultaneously. In particular, the bandwidth requirements for a bunch-by-bunch feedback system can be reduced considerably.

A similar argument holds for the proposed proton driver, while this method might also be applied to the Main Injector, in particular when higher proton currents are reached where coupled-bunch oscillations might cause significant emittance dilution. 


\section{References}

[1] D. Moehl, CERN Report MPS/DL/70-09 (1970);

[2] D. Boussard, J. Gareyte, 8-th Internat. Conf.on High-Energy Accelerators, CERN (1971), p.317;

[3] R. Kohaupt, DESY Report HI/74/2 (1974) and 4-th All-Union Conf., Sowiet Union (1975), p.349;

[4] C. Ankenbrandt et al, Trans.IEEE-NS-24 (1977) p.1449;

[5] D. Moehl, LBL Report (1972);

[6] Y. Chin, K. Yokoya, DESY Report 86-097(1986);

[7] R. Kohaupt, DESY Report 86-121 (1986);

[8] I. Gradshteyn, I. Rhyzik, Table of Integrals, Series and Products, Acad. Press 1980;

[9] J. Laslett et al, Rev. Sci. Instr.36 (1965) p.436;

[10] K.Y. Ng in Fermilab Report TM-2169 (2002) p.4-7;.

\section{Disclaimer}

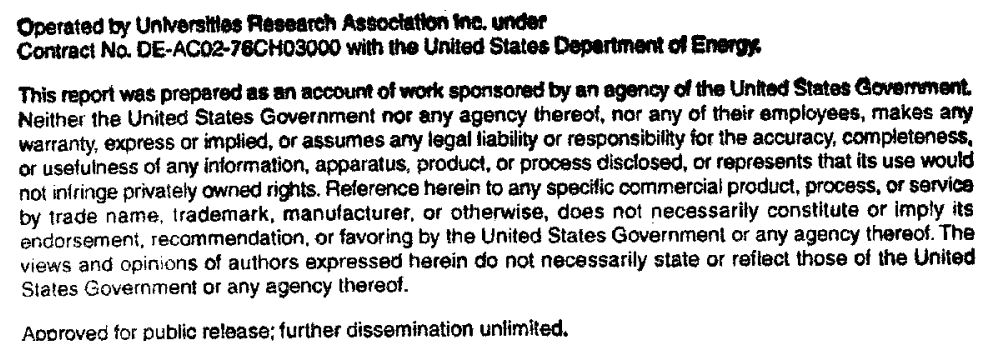

Approved tor public reloase; further dissemination unlimited. 


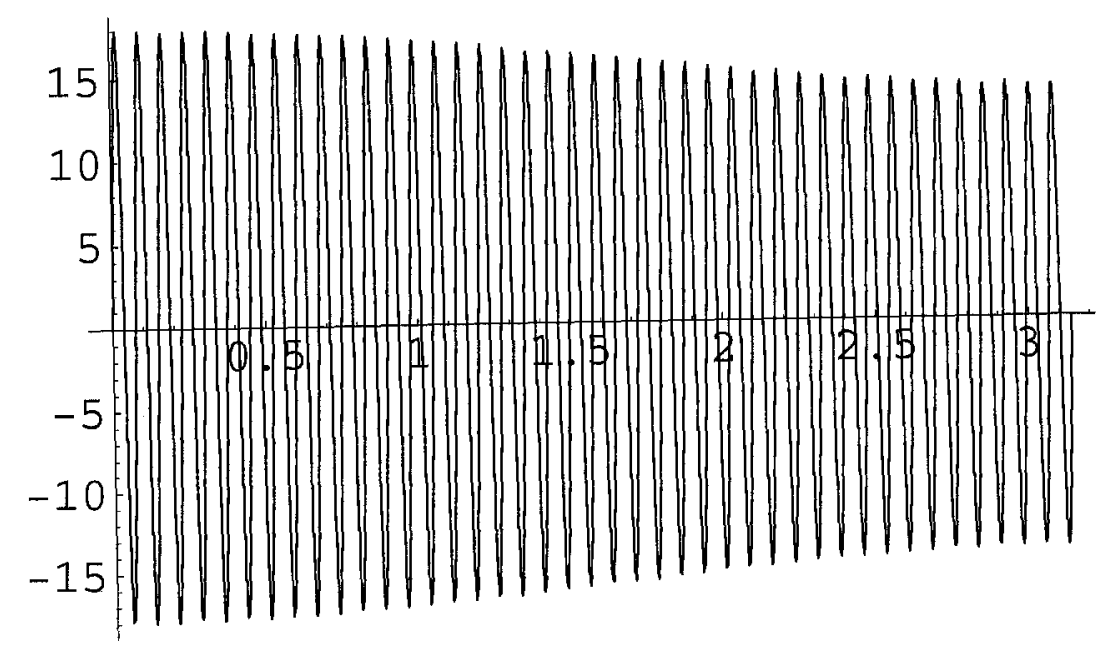

Figure 1: Rf voltage (arbitrary units) versus time for one half revolution period, tuning 2 of $18 \mathrm{rf}$ cavities down from 84-th to 83-rd harmonic.

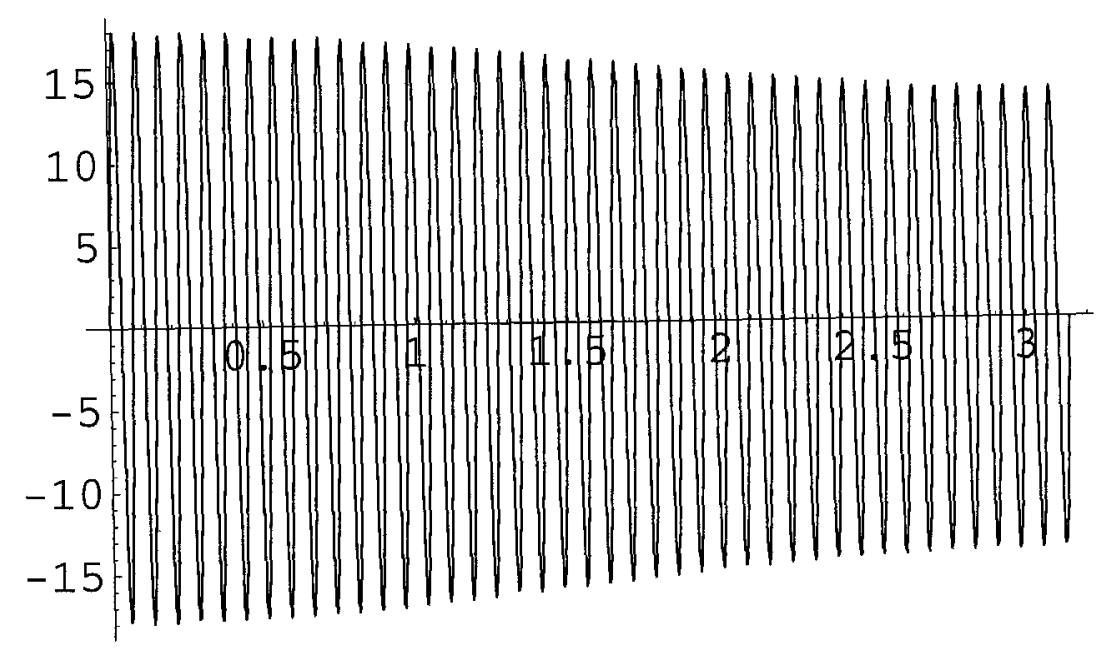

Figure 2: Rf voltage (arbitrary units) versus time, tuning 1 of 18 rf cavities from 84-th harmonic down to 83-rd, and 1 up to 85-th harmonic. 